\title{
A Suggested Method for Evaluation of Sandalwood Essential oil Quality
}

\section{Dinh Xuan Ba*}

Associate Professor, Director of SECOIN Applied Biology Center, Vietnam

*Corresponding Author: Dinh Xuan Ba, Associate Professor, Director of SECOIN Applied Biology Center, Vietnam.

Received: August 14, 2019; Published: August 19, 2019

DOI: 10.31080/ASAG.2019.03.0622

Assume that we have Sandalwood essential oil extracted by hydrodistillation technique or by a modified hydrodistillation, for example Microwave HydroDistillation (MHD) or Microwave AirHydro Distillation (MAHD) [1]. After making GC-MS analysis on this Sandalwood essential oil we have a data set of volatile and semi-volatile compounds. From these compounds we should pay attention to the following entities:

Let $\mathrm{s}$ be the total content of all sesquiterpenes $\left(\mathrm{C}_{15} \mathrm{H}_{24}\right)$ and their derivatives

Let $\mathrm{s}_{\mathrm{o}}$ be the total content of all oxygenated sesquiterpenes and their derivatives

Let $\mathrm{m}$ be the total content of all monoterpenes $\left(\mathrm{C}_{10} \mathrm{H}_{14}, \mathrm{C}_{10} \mathrm{H}_{16}\right)$ and their derivatives

Let $\mathrm{m}_{\mathrm{o}}$ be the total content of all oxygenated monoterpenes and their derivatives

Let e be the total content of all fatty acids and their derivatives

Let $\mathrm{f}$ be the total content of all allergens

Let $g$ be the total content of all toxins

Let $\mathrm{S}$ be the total content of all compouds listed in the GC-MS Test Report

Similar to our previous finding [2], we first derive from experiences and sensory comments.

Practical experiences and intuitional consideration

The higher $\mathrm{s}_{\mathrm{o}}$ (in comparison with s) the better aromatic scent and therapeutic effects of sandalwood essential oil [3]. The smaller m (in comparison with s) the better quality of sandalwood essential oil. For a normal sandalwood essential oil, we obviously require: $e$ is small, $s+s_{o}$ is big, $f=g=0$ and $S \geq 75 \%$.

We hereby propose a suggested method for evaluation of sandalwood essential oil quality (in chemical composition):

Let $\alpha=\frac{\mathrm{s}_{\circ}+\mathrm{m}_{\mathrm{o}}}{\mathrm{s}+\mathrm{m}}$ then the bigger the $\alpha$, the higher the quality of sandalwood essential oil on condition that $\beta=s+s o \geq 65 \%$ (this constrain of $\beta$ is also mentioned in [2]. This criteria can applicable to agarwood essential oil (not only to sandalwood essential oil).
We try to apply $\alpha$ to evaluate the quality of essential oils mentioned in the following article: H.S. Kusuma and M. Mahfud [1] extracted sandalwood (santalum album) essential oils from different techniques: Microwave hydrodistillation (MHD), see figure 1, and Microwave air-hydrodistillation (MAHD), see figure 2. After making GC-MS analysis they obtained two sets of data, each set consists of 59 compound names together with their corresponding content (\%). Sum of 59 contents $\mathrm{S}=100 \%$. From these two sets of data we extracted a list of monoterpenes and oxygenated monoterpenes, see table 1 , and a list of sesquiterpenes and oxygenated sesquiterpenes, see table 2 .

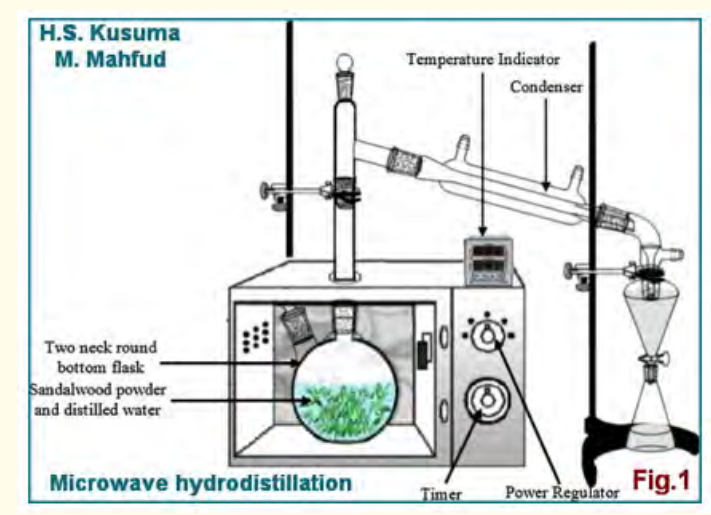

Figure 1

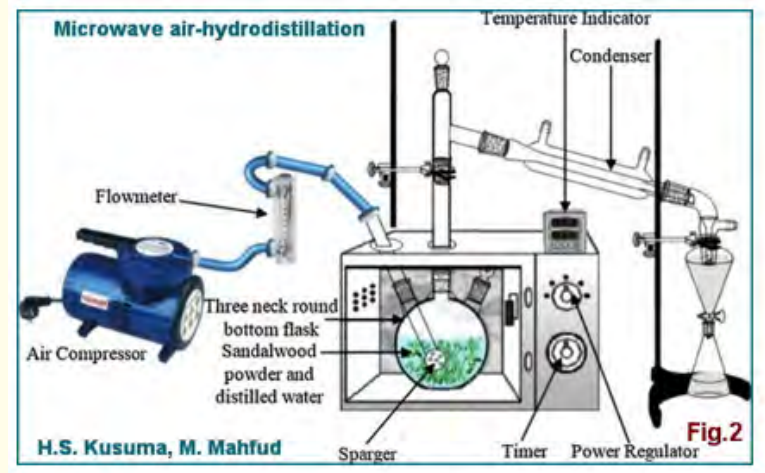

Figure 2 


\begin{tabular}{|l|l|c|c|c|c|}
\hline \multirow{2}{*}{ No } & Compound name & Molecular & \multirow{2}{*}{ Classification } & \multicolumn{2}{|c|}{ Content (\%) } \\
\cline { 4 - 6 } & & Formula & & MHD & MAHD \\
\hline 1 & Camphene & $\mathrm{C}_{10} \mathrm{H}_{16}$ & monoterpene & 0.95 & 0.82 \\
\hline 2 & Alpha-Pinene & $\mathrm{C}_{10} \mathrm{H}_{16}$ & monoterpene & 0.06 & $\mathrm{nd}$ \\
\hline 3 & Santolina triene & $\mathrm{C}_{10} \mathrm{H}_{16}$ & monoterpene & 0.07 & $\mathrm{nd}$ \\
\hline 4 & Beta-ocimene & $\mathrm{C}_{10} \mathrm{H}_{16}$ & monoterpene & 0.58 & $\mathrm{nd}$ \\
\hline 5 & cis-ocimene & $\mathrm{C}_{10} \mathrm{H}_{16}$ & monoterpene & 1.87 & 0.09 \\
\hline 6 & (Z)-alloocimene & $\mathrm{C}_{10} \mathrm{H}_{16}$ & monoterpene & 0.06 & $\mathrm{nd}$ \\
\hline 7 & Alpha-terpinene & $\mathrm{C}_{10} \mathrm{H}_{16}$ & monoterpene & 1.04 & 1.11 \\
\hline 8 & 1-cyclohexylidene-2-methylpropene & $\mathrm{C}_{10} \mathrm{H}_{16}$ & monoterpene & $\mathrm{nd}$ & 1.19 \\
\hline 9 & Isoterpinolene & $\mathrm{C}_{10} \mathrm{H}_{16}$ & monoterpene & 0.31 & $\mathrm{nd}$ \\
\hline & Sum & & m & $\mathbf{4 . 9 4}$ & $\mathbf{3 . 2 1}$ \\
\hline 1 & 8-methylene-2-exo-noradamantanol & $\mathrm{C}_{10} \mathrm{H}_{14} \mathrm{O}$ & Oxygenated monoterpene & $\mathrm{nd}$ & 0.04 \\
\hline 2 & $\begin{array}{l}\text { 3a,6-methano-3aH-inden-7(4H)-one, } \\
\text { hexahydro-7a-d-, (3aa,6a,7ab)- }\end{array}$ & $\mathrm{C}_{10} \mathrm{H}_{14} \mathrm{O}$ & Oxygenated monoterpene & 0.72 & $\mathrm{nd}$ \\
\hline 3 & Teresantalol & $\mathrm{C}_{10} \mathrm{H}_{16} \mathrm{O}$ & Oxygenated monoterpene & 4.58 & 1.45 \\
\hline 4 & Sabinene hydrate & $\mathrm{C}_{10} \mathrm{H}_{18} \mathrm{O}$ & Oxygenated monoterpene & 0.10 & nd \\
\hline & Sum & & $\mathbf{5 . 4}$ & $\mathbf{1 . 4 9}$ \\
\hline
\end{tabular}

Table 1: List of monoterpenes and oxygenated monoterpenes. nd: not detected

\begin{tabular}{|l|l|c|c|c|c|}
\hline \multirow{2}{*}{ No } & Compound name & Molecular & Classification & \multicolumn{2}{|c|}{ Content (\%) } \\
\cline { 3 - 5 } & & Formula & & MHD & MAHD \\
\hline 1 & Alpha-farnesene & $\mathrm{C}_{15} \mathrm{H}_{24}$ & Sesquiterpene & 0.50 & 1.88 \\
\hline 2 & Beta-patchoulene & $\mathrm{C}_{15} \mathrm{H}_{24}$ & Sesquiterpene & 0.62 & nd \\
\hline 3 & Alpha-santalene & $\mathrm{C}_{15} \mathrm{H}_{24}$ & Sesquiterpene & nd & 0.03 \\
\hline 4 & Alpha-cedrene & $\mathrm{C}_{15} \mathrm{H}_{24}$ & Sesquiterpene & nd & 0.05 \\
\hline 5 & Gamma-curcumene & $\mathrm{C}_{15} \mathrm{H}_{24}$ & Sesquiterpene & nd & 0.17 \\
\hline 6 & Alpha-guaiene & $\mathrm{C}_{15} \mathrm{H}_{24}$ & Sesquiterpene & 0.66 & 0.11 \\
\hline 7 & Isosativene & $\mathrm{C}_{15} \mathrm{H}_{24}$ & Sesquiterpene & 0.18 & nd \\
\hline 8 & Beta-santalene & $\mathrm{C}_{15} \mathrm{H}_{24}$ & Sesquiterpene & 0.05 & 0.07 \\
\hline 9 & Seychellene & $\mathrm{C}_{15} \mathrm{H}_{24}$ & Sesquiterpene & 0.77 & nd \\
\hline 10 & Alpha-patchoulene & $\mathrm{C}_{15} \mathrm{H}_{24}$ & Sesquiterpene & 0.38 & nd \\
\hline 11 & Germacrene B & $\mathrm{C}_{15} \mathrm{H}_{24}$ & Sesquiterpene & 5.50 & 0.10 \\
\hline 12 & alpha-selinene & $\mathrm{C}_{15} \mathrm{H}_{24}$ & Sesquiterpene & 0.06 & $\mathrm{nd}$ \\
\hline 13 & Delta-guaiene & $\mathrm{C}_{15} \mathrm{H}_{24}$ & Sesquiterpene & 0.48 & 0.15 \\
\hline 14 & Gamma-elemene & $\mathrm{C}_{15} \mathrm{H}_{24}$ & Sesquiterpene & nd & 3.69 \\
\hline 15 & Alpha-bergamotene & $\mathrm{C}_{15} \mathrm{H}_{24}$ & Sesquiterpene & nd & 0.20 \\
\hline 16 & Bicyclogermacrene & $\mathrm{C}_{15} \mathrm{H}_{24}$ & Sesquiterpene & 0.64 & 0.45 \\
\hline 17 & 2-methyl-6-(4-methylcyclohex-3-en- & $\mathrm{C}_{15} \mathrm{H}_{24}$ & Sesquiterpene & nd & 0.71 \\
\hline 18 & Acoradiene & $\mathrm{C}_{15} \mathrm{H}_{24}$ & Sesquiterpene & 1.39 & 0.59 \\
\hline 19 & Bicycloelemene & $\mathrm{C}_{15} \mathrm{H}_{24}$ & Sesquiterpene & nd & 1.36 \\
\hline
\end{tabular}




\begin{tabular}{|l|l|c|c|c|c|}
\hline 20 & Viridiflorene (ledene) & $\mathrm{C}_{15} \mathrm{H}_{24}$ & Sesquiterpene & 0.91 & nd \\
\hline & Sum & & $\mathbf{1}$ & $\mathbf{1 2 . 1 4}$ & $\mathbf{9 . 5 6}$ \\
\hline 1 & Beta-santalol & $\mathrm{C}_{15} \mathrm{H}_{24} \mathrm{O}$ & Oxygenated sesquiterpene & 22.67 & 24.80 \\
\hline 2 & Alpha-santalol & $\mathrm{C}_{15} \mathrm{H}_{24} \mathrm{O}$ & Oxygenated sesquiterpene & 27.81 & 28.73 \\
\hline 3 & Alpha-bergamotol & $\mathrm{C}_{15} \mathrm{H}_{24} \mathrm{O}$ & Oxygenated sesquiterpene & 10.82 & 10.18 \\
\hline 4 & cis-alpha-copaene-8-ol & $\mathrm{C}_{15} \mathrm{H}_{24} \mathrm{O}$ & Oxygenated sesquiterpene & 1.86 & $\mathrm{nd}$ \\
\hline 5 & cis-lanceol & $\mathrm{C}_{15} \mathrm{H}_{24} \mathrm{O}$ & Oxygenated sesquiterpene & 3.42 & 2.74 \\
\hline 6 & Alpha-cedrol & $\mathrm{C}_{15} \mathrm{H}_{26} \mathrm{O}$ & Oxygenated sesquiterpene & $\mathrm{nd}$ & 2.65 \\
\hline 7 & Caryophylla-3,8(15)-dien-5alpha-ol & $\mathrm{C}_{15} \mathrm{H}_{26} \mathrm{O}$ & Oxygenated sesquiterpene & 0.47 & 0.52 \\
\hline 8 & Isolongifolol & $\mathrm{C}_{15} \mathrm{H}_{26} \mathrm{O}$ & Oxygenated sesquiterpene & 0.21 & 0.59 \\
\hline & Sum & & $\mathbf{6 7}$ & $\mathbf{6 7 . 2 6}$ & $\mathbf{7 0 . 2 1}$ \\
\hline
\end{tabular}

Table 2: List of sesquiterpenes and oxygenated sesquiterpenes.

nd: not detected

So, we have

\begin{tabular}{|l|c|c|}
\hline & For MHD & For MAHD \\
\hline$\beta=\mathrm{s}+\mathrm{s}_{\mathrm{o}}$ & $67.26 \%+12.14 \%=79.40 \%$ & $70.21 \%+9.56 \%=79.77 \%$ \\
\hline$\alpha=\frac{\mathrm{s}_{\mathrm{o}}+\mathrm{m}_{0}}{\mathrm{~s}+\mathrm{m}}$ & $=4.254$ & $=5.615$ \\
& & \\
\hline
\end{tabular}

\section{Conclusion}

MAHD technique is (chemically) better than MHD technique.

Usually we want both $\alpha$ and $\beta$ to be large, but in practice when $\beta$ grows (for example when $s>$ so), it makes the $\alpha$ smaller, then we need to choose an acceptable compromise of $\alpha$ and $\beta$. For a possible compromise between $\alpha$ and $\beta$ we can choose two positive numbers $\mathrm{x}$ and $\mathrm{y},(\mathrm{x}+\mathrm{y}=1)$, for forming the function $\omega=\mathrm{x}^{*} \alpha+\mathrm{y}^{*} \beta$. So, the bigger $\omega$ the better (chemically) quality of sandalwood essential oil.

\section{Bibliography}

1. HS Kusuma and M Mahfud. "The Extraction of Essential Oil from Sandalwood (Santalum album) by Microwave Air-Hydrodistillation Method". Journal of Materials and Environmental Science 7.5 (2016): 1597-1606.
2. Dinh xuan Ba. "Suggested method for evaluation of agarwood oil quality". International Journal of Agricultural Research and Reviews 56 (2017): 644-646.

3. Michael Zviely and Ming Li. Sesquiterpenoids: The Holy Fragrance Ingredients. The Key Laboratory of Food Colloids and Biotechnology, Ministry of Education, Department of Applied Chemistry, School of Chemical and Material Engineering, Jiang Nan University, China.

\section{Volume 3 Issue 9 September 2019}

(C) All rights are reserved by Dinh Xuan Ba. 